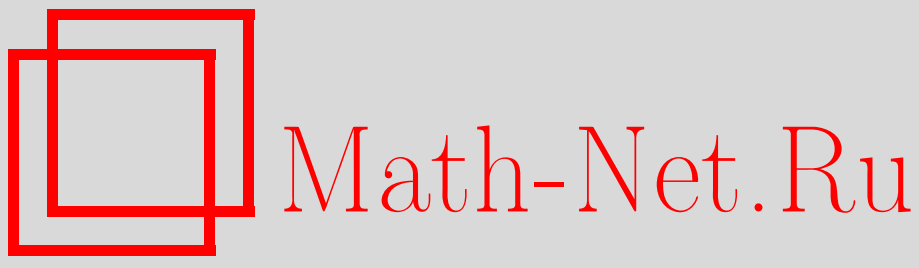

В. С. Климов, Функциональные неравенства и обобщенные емкости, Матем. сб., 1996, том 187, номер 1, 41-54

DOI: https://doi.org/10.4213/sm99

Использование Общероссийского математического портала Math-Net.Ru подразумевает, что вы прочитали и согласны с пользовательским соглашением

http: //www. mathnet.ru/rus/agreement

Параметры загрузки:

IP : 52.205 .19 .152

26 апреля 2023 г., 15:51:21 
УДК 517.518.235

\author{
В. С. Климов
}

\title{
Функциональные неравенства и обобщенные емкости
}

В статье найдены критерии справедливости функционального неравенства вида $\|f ; Q\| \leqslant C\|\nabla f ; P\|$, где $P, Q$ - нормированные идеальные пространства функций на области $\Omega \subset \mathbb{R}^{n}$, константа $C$ одинакова для удовлетворяющих условию Липшица финитных функций $f$. Приведены условия согласования норм в пространствах $P, Q$, при вьполнении которых исследуемое функциональное неравенство равносильно геометрическому неравенству, связывающему $Q$-нормы индикаторов и $P$-емкости компактных подмножеств области $\Omega$. Даны оценки и изучены общие свойства емкостей.

Библиограбоия: 20 названий.

В статье найдены критерии справедливости функционального неравенства вида

$$
\|f ; Q\| \leqslant C\|\nabla f ; P\|,
$$

где $P, Q$ - нормированные идеальные пространства функций на области $\Omega \subset \mathbb{R}^{n}$, константа $C$ одинакова для всех финитных функций $f$, удовлетворяющих условию Липшица. В предположении некоторого согласования норм в пространствах $P, Q$ неравенство (1) равносильно геометрическому неравенству, связывающему $Q$-нормы индикаторов и $P$-емкости компактных подмножеств $\Omega$. Приведены условия согласования соответствующих норм, даны оценки и изучены общие свойства емкостей. Основное внимание уделено случаю, когда $P$ совпадает с пространством Орлича.

Для $P=L^{p}\left(\Omega, \mathbb{R}^{n}\right), Q=L^{q}(\Omega)$ аналогичные результаты получены ранее в работах В.Г. Мазья [1], [2]. Основное содержание данной статьи связано с распространением результатов В. Г. Мазья на более общие функциональные пространства. С этой целью введено и изучено понятие мажорирования $Q \prec P$, выражающее некоторое условие согласования норм в идеальных пространствах $P, Q$ и связанное с определениями $\sigma$-супераддитивности и $\sigma$-субаддитивности (см. ниже).

1. Пусть $(X, \mu)$ - измеримое пространство с полной $\sigma$-конечной непрерьвной мерой $\mu ; \mathbb{R}^{n}-n$-мерное евклидово пространство над полем $\mathbb{R}$ действительных чисел со скалярным произведением $a \cdot b$ и нормой $|a|=\sqrt{a \cdot a}\left(a, b \in \mathbb{R}^{n}\right) ; S\left(X, \mathbb{R}^{n}\right)-$ линейное метрическое пространство $\mu$ п.в. (почти всюду) конечных $\mu$-измеримых функций на $X$ со значениями в $\mathbb{R}^{n}$. Пространство $S(X, \mathbb{R})$ обозначается далее через $S(X)$, аналогичные сокрашения используются и для других пространств скалярных функций.

Линейное пространство $E=E\left(X, \mathbb{R}^{n}\right)$ функций класса $S\left(X, \mathbb{R}^{n}\right)$ называют нормированным идеальным пространством (НИП), если из соотношений $y \in E$, $\alpha \in S(X),|\alpha(x)| \leqslant 1$ п.в. следует, что $\alpha y \in E$ и $\|\alpha y ; E\| \leqslant\|y ; E\|$. Если НИП

$$
\text { (C) В.С. Климов } 1996
$$


$E$ полно относительно нормы $\|\cdot ; E\|$, то его назьвают банаховым идеальным пространством (БИП). Теории БИП и связанному с ней выпуклому анализу посвящены многие публикации (см., например, работы [3]-[11] и приведенную в них библиографиюю).

Опишем некоторые способы конструирования НИП. Обозначим через $\beta\left(\mathbb{R}^{n}\right)$ класс выпуклых [9] четных функций $\Phi: \mathbb{R}^{n} \rightarrow \mathbb{R}, \Phi(0)=0, \Phi(\xi)>0$ при $\xi \neq 0$. Пусть $H=H(X)$ - НИП скалярных функций. Сопоставим $H$ и функции $\Psi$ из $\beta\left(\mathbb{R}^{n}\right)$ совокупность функций класса $S\left(X, \mathbb{R}^{n}\right)$, для которых имеет смысл и конечна норма

$$
\left\|y ; H^{\Psi}\right\|=\inf \left\{k>0:\left\|\Psi\left(\frac{y}{k}\right) ; H\right\| \leqslant 1\right\} .
$$

Как нетрудно проверить, $H^{\Psi}\left(X, \mathbb{R}^{n}\right)$ есть НИП. Если $H=L^{1}(X)$ - пространство суммируемых по мере $\mu$ функций, то $H^{\Psi}\left(X, \mathbb{R}^{n}\right)$ обозначают символом $L^{\Psi}\left(X, \mathbb{R}^{n}\right)$ и называют пространством Орлича; соответствующая норма $\left\|\cdot ; L^{\Psi}\right\|$ совпадает с нормой Люксембурга [3, с. 150], [10, с. 95], [11, с. 202].

Пусть $F=F\left(X, \mathbb{R}^{n}\right)$ есть НИП, $\psi:[0, \infty) \rightarrow[0, \infty)$ - квазивогнутая $[4$, с. 70$]$ функция. Обозначим через $F_{\psi}=F_{\psi}\left(X, \mathbb{R}^{n}\right)$ часть $S\left(X, \mathbb{R}^{n}\right)$, состояшую из функций $y$, для которых имеет смысл и конечна норма

$$
\left\|y ; F_{\psi}\right\|=\sup \left\{\frac{1}{\psi(\mu(D))}\left\|1_{D} y ; F\right\|, 0<\mu(D)<\infty\right\}
$$

здесь и далее $1_{D}$ - индикатор (характеристическая функция) множества $D$. Ecли $F=L^{1}\left(X, \mathbb{R}^{n}\right)$, то $F_{\psi}$ совпадает с пространством Марцинкевича $M_{\psi}\left(X, \mathbb{R}^{n}\right)$ [4, с. 154$]$. НИП $H^{\Psi}\left(X, \mathbb{R}^{n}\right), F_{\psi}\left(X, \mathbb{R}^{n}\right)$ будут далее использоваться в ряде иллюстрируюших примеров.

Пусть $\mathbb{Z}$ - множество целых чисел. Банахово пространство $\sigma=\sigma(\mathbb{Z})$ последовательностей $a=\left(a_{i}\right)(i \in \mathbb{Z})$ называют [4, с. 213] симметричньм, если из условий $a=\left(a_{i}\right) \in \sigma,\left|b_{i}\right| \leqslant\left|a_{h(i)}\right|, b=\left(b_{i}\right)(i \in \mathbb{Z}, h-$ биекция множества $\mathbb{Z})$ следует, что $b \in \sigma$ и $\|b ; \sigma\| \leqslant\|a ; \sigma\|$. Через $\ell_{M}=\ell_{M}(\mathbb{Z})$ ниже обозначается пространство последовательностей $a=\left(a_{i}\right)(i \in \mathbb{Z})$, порожденное выпуклой четной непрерывной в нуле функцией $M: \mathbb{R} \rightarrow[0, \infty]=\overline{\mathbb{R}}_{+}, M(0)=0, M(s)>0$ при $s>0$, с нормой

$$
\left\|a ; \ell_{M}\right\|=\inf \left\{k>0, \sum_{i \in \mathbb{Z}} M\left(\frac{a_{i}}{k}\right) \leqslant 1\right\} .
$$

Пространство $\ell_{M}=\ell_{M}(\mathbb{Z})$ симметрично; при $M(s)=|s|^{p}(1 \leqslant p<\infty)$ оно обозначается символом $\ell_{p}=\ell_{p}(\mathbb{Z})$.

Функциональную последовательность $y_{i}$ из $S\left(X, \mathbb{R}^{n}\right)(i \in \mathbb{Z})$ назовем $\partial и з б-$ юнктной, если $\left|y_{i}(x)\right|\left|y_{j}(x)\right|=0 \quad \mu$ п.в. при $i \neq j$. Пусть $\sigma=\sigma(\mathbb{Z})$ - симметричное пространство $(\mathrm{C \Pi})$. НИП $E=E\left(X, \mathbb{R}^{n}\right)$ назовем $\sigma$-супераддитивныц. $\left(\sigma\right.$-субаддитивныл), если для каждой дизъюнктной последовательности $y_{i} \in E$ $(i \in \mathbb{Z})$ имеет место оценка $\|y ; E\| \geqslant\|a ; \sigma\|$ (соответственно, $\|y ; E\| \leqslant\|a ; \sigma\|)$, где

$$
a=\left(a_{i}\right), \quad a_{i}=\left\|y_{i} ; E\right\|(i \in \mathbb{Z}), \quad y=\sum_{i \in \mathbb{Z}} y_{i} ;
$$


при этом считается, что если $y \notin E$, то $\|y ; E\|=\infty$, аналогичное соглашение относится и к $\|a ; \sigma\|$.

Пространство $L^{p}\left(X, \mathbb{R}^{n}\right)(1 \leqslant p<\infty)$ одновременно $\ell_{p}$-супераддитивно и $\ell_{p}$-субаддитивно. В случае $\sigma=\ell_{p}(1 \leqslant p<\infty)$ понятия, близкие к $\sigma$-субаддитивности и $\sigma$-супераддитивности, вводились многими авторами (библиография может быть найдена в обзорах [5], [6]).

Пусть $P=P\left(X, \mathbb{R}^{n}\right), Q=Q\left(X_{1}, \mathbb{R}^{m}\right)$ два НИП. Скажем, что $P$ мажорирует $Q(Q \prec P)$, если существуют такие симметричные пространства $\sigma=\sigma(\mathbb{Z})$, $\tau=\tau(\mathbb{Z})$, что $P-\sigma$-супераддитивное пространство, $Q-\tau$-субаддитивное пространство и $\sigma$ непрерьвно вложено в $\tau$. Точная нижняя грань норм операторов вложения $J: \sigma \rightarrow \tau$, вычисляемая по всем подобным парам $\sigma, \tau$, обозначается символом $\nu(P, Q)$. Требование $Q \prec P$ выражает определенную согласованность норм в пространствах $P, Q$. Константа $\nu(P, Q)$ в некотором смысле характеризует степень этой согласованности.

2. Приведем несколько иллюстрирующих примеров.

Лемма 1. Пусть $\Phi \in \beta\left(\mathbb{R}^{n}\right), M: \mathbb{R} \rightarrow \overline{\mathbb{R}}_{+}-$вылуклая четная функиия, $M(0)=0, M(s)>0 \quad(s>0), M(1)=1 u$

$$
\Phi(t \xi) \geqslant \Phi(\xi) M(t) \quad\left(\xi \in \mathbb{R}^{n}, 0 \leqslant t \leqslant 1\right) .
$$

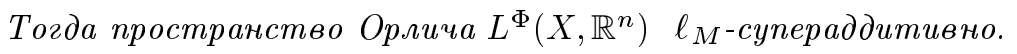

ДокАЗАТЕЛЬство. Пусть $y_{i}(i \in \mathbb{Z})$ - дизъюнктная последовательность элементов пространства $L^{\Phi}\left(X, \mathbb{R}^{n}\right)$,

$$
a_{i}=\left\|y_{i} ; L^{\Phi}\right\|, \quad a=\left(a_{i}\right), \quad y=\sum_{i \in \mathbb{Z}} y_{i}, \quad\left\|y ; L^{\Phi}\right\|<1 .
$$

Из условий леммы легко выводится непрерьвность на $L^{\Phi}\left(X, \mathbb{R}^{n}\right)$ функционала $I_{\Phi}(\cdot)=\left\|\Phi(\cdot) ; L^{1}\right\|$. В частности, если $a_{i}>0$, то $I_{\Phi}\left(y_{i} / a_{i}\right)=1$. Ввиду этого имеем

$$
1 \geqslant I_{\Phi}(y)=\sum_{i \in \mathbb{Z}} I_{\Phi}\left(y_{i}\right) \geqslant \sum_{a_{i}>0} I_{\Phi}\left(\frac{y_{i}}{a_{i}}\right) M\left(a_{i}\right)=\sum_{i \in \mathbb{Z}} M\left(a_{i}\right) .
$$

Следовательно, $\left\|a ; \ell_{M}\right\| \leqslant 1$ как только $\left\|y ; L^{\Phi}\right\|<1$, а это означает $\ell_{M^{-}}$супераддитивность пространства Орлича $L^{\Phi}\left(X, \mathbb{R}^{n}\right)$. Лемма доказана.

Лемма 2. Пусть пространство $H=H(X)$ есть БИП. $\Psi \in \beta\left(\mathbb{R}^{n}\right), M(t)=$ $\sup \{\Psi(t \xi) / \Psi(\xi), \xi \neq 0\}$. Тогда пространство $H^{\Psi}\left(X, \mathbb{R}^{n}\right) \quad \ell_{M}$-субаддитивно.

ДокАЗАТЕЛьство. Пусть $y_{i}, a_{i}, y, a$ имеют тот же смысл, что и в лемме 1 , причем $\left\|a ; \ell_{M}\right\|<1$. Если $0<\lambda<1$, то

$$
\begin{aligned}
\|\Psi(\lambda y) ; H\| & =\left\|\sum_{a_{i}>0} \Psi\left(a_{i} \frac{\lambda y_{i}}{a_{i}}\right) ; H\right\| \\
& \leqslant \sum_{a_{i}>0} M\left(a_{i}\right)\left\|\Psi\left(\lambda \frac{y_{i}}{a_{i}}\right) ; H\right\| \leqslant \sum_{i \in \mathbb{Z}} M\left(a_{i}\right)<1 .
\end{aligned}
$$


Таким образом, $\left\|y ; H^{\Psi}\right\| \leqslant 1$, если только $\left\|a ; \ell_{M}\right\|<1$, а это влечет $\ell_{M^{-c y б-~}}$ аддитивность пространства $H^{\Psi}$. Лемма доказана.

Двойственныцм к СП $\sigma=\sigma(\mathbb{Z})$ называют пространство $\sigma^{\prime}=\sigma^{\prime}(\mathbb{Z})$, состояшее из таких последовательностей $b=\left(b_{i}\right), i \in \mathbb{Z}$, для которых имеет смысл и конечна норма $\left\|b ; \sigma^{\prime}\right\|=\sup \{a \cdot b,\|a ; \sigma\| \leqslant 1\}$, где $a \cdot b=\sum a_{i} b_{i}(i \in \mathbb{Z})$. Двойственное к пространству Орлича $\ell_{N}=\ell_{N}(\mathbb{Z})$ совпадает с пространством Орлича $(\ell)_{N^{*}}$, порожденным сопряженной к $N$ функцией $N^{*}[9$, с. 120$]$ и наделенным так назьваемой нормой Орлича $\left\|b ;(\ell)_{N^{*}}\right\|=\sup \left\{a \cdot b,\left\|a ; \ell_{N}\right\| \leqslant 1\right\}$.

Аналогично, но несколько более сложно, НИП $E=E\left(X, \mathbb{R}^{n}\right)$ можно сопоставить двойственное к нему $E^{\prime}=E^{\prime}\left(X, \mathbb{R}^{n}\right)[8]$. Пространство $E^{\prime}$ всегда полно. Если $y \in E, z \in E^{\prime}$, то [8]

$$
\langle y, z\rangle=\int_{X} y(x) \cdot z(x) d \mu(x)<\infty, \quad\left\|z ; E^{\prime}\right\|=\sup \{\langle y, z\rangle,\|y ; E\| \leqslant 1\} .
$$

Далее будут использоваться вытекающие из определений двойственных пространств варианты неравенства Гёльдера

$$
a \cdot b \leqslant\|a ; \sigma\|\left\|b ; \sigma^{\prime}\right\|, \quad\langle y, z\rangle \leqslant\|y ; E\|\left\|z ; E^{\prime}\right\|,
$$

где $a \in \sigma, b \in \sigma^{\prime}, y \in E, z \in E^{\prime}$.

Лемма 3. Пусть $F=F\left(X, \mathbb{R}^{n}\right)$ есть БИП, $\psi:[0, \infty) \rightarrow[0, \infty)$ - квазивогнутая функция. Пусть $(\ell)_{N^{*}}-$ пространство, двойственное к пространству Орлича $\ell_{N}$. Eсли $\psi^{-1}(t s) \geqslant \psi^{-1}(s) N(t) \quad(s \geqslant 0,0 \leqslant t \leqslant 1)$, то пространство

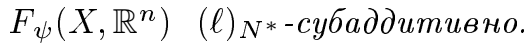

ДокАЗАТЕЛЬство. Пусть $y_{i}, a_{i}, y, a$ определены так же, как и в леммах 1,2 ; в частности, $a_{i}=\left\|y_{i} ; F_{\psi}\right\|(i \in \mathbb{Z}), a=\left(a_{i}\right)$. Обозначим через $X_{i}$ носитель $\operatorname{supp}\left|y_{i}\right|$ функции $\left|y_{i}\right|[3$, c. 137]. Фиксируем измеримое множество $D(0<\mu(D)<\infty)$ и положим $s=\psi(\mu(D)), t_{i}=\psi\left(\mu\left(D \cap X_{i}\right)\right) / s(i \in \mathbb{Z})$. Очевидно, что $\mu(D)=\psi^{-1}(s)$, $\mu\left(D \cap X_{i}\right)=\psi^{-1}\left(t_{i} s\right)$ и

$$
\left\|1_{D} y_{i} ; F\right\| \leqslant a_{i} \psi\left(\mu\left(D \cap X_{i}\right)\right)=a_{i} t_{i} s \quad(i \in \mathbb{Z}),
$$

следовательно,

$$
\left\|1_{D} y ; F\right\| \leqslant \sum_{i \in \mathbb{Z}}\left\|1_{D} y_{i} ; F\right\| \leqslant s \sum_{i \in \mathbb{Z}} a_{i} t_{i} .
$$

Поскольку $N\left(t_{i}\right) \leqslant \psi^{-1}\left(t_{i} s\right) / \psi^{-1}(s)=\mu\left(D \cap X_{i}\right) / \mu(D)$, то

$$
\sum_{i \in \mathbb{Z}} N\left(t_{i}\right) \leqslant \sum_{i \in \mathbb{Z}} \mu\left(D \cap X_{i}\right) / \mu(D) \leqslant 1 .
$$

Таким образом, $t=\left(t_{i}\right) \in \ell_{N}$ и $\left\|t ; \ell_{N}\right\| \leqslant 1$.

Объединяя установленные выше оценки, получаем

$$
\left\|1_{D} y ; F\right\| \leqslant s a \cdot t \leqslant s\left\|a ;(\ell)_{N^{*}}\right\|\left\|t ; \ell_{N}\right\| \leqslant\left\|a ;(\ell)_{N^{*}}\right\| \psi(\mu(D)),
$$

т.е. $\left\|y ; F_{\psi}\right\| \leqslant\left\|a ;(\ell)_{N^{*}}\right\|$. Лемма доказана. 
Лемма 4. Пусть $\varphi:[0, \infty) \rightarrow[0, \infty)$ - возрастающая строго вогнутая функиия, $\varphi(0)=0$,

$$
\psi(t)=\frac{t}{\varphi(t)}(t>0), \quad \psi(0)=0, \quad M(t)=\sup \left\{\frac{\varphi^{-1}(|t| s)}{\varphi^{-1}(s)}, s>0\right\}
$$

Тогда для любого БИП $F=F\left(X, \mathbb{R}^{n}\right)$ пространство $F_{\psi}\left(X, \mathbb{R}^{n}\right) \quad(\ell)_{M^{-} \text {-суб- }}$ аддитивно.

ДоказАтельство. Пусть $\Phi:[0, \infty) \rightarrow[0, \infty)$ - функция, обратная к $\varphi$. В силу определения функции $M$ выполняется неравенство $\Phi(t s) \leqslant \Phi(s) M(t)(s \geqslant 0$, $0 \leqslant t \leqslant 1)$. Положим $M(t)=t_{1}, \Phi(s)=s_{1}$. Тогда последнее неравенство влечет оценку $M^{-1}\left(t_{1}\right) \leqslant \varphi\left(t_{1} s_{1}\right) / \varphi\left(s_{1}\right)\left(s_{1}>0,0 \leqslant t_{1} \leqslant 1\right)$. Так как $\left(M^{*}\right)^{-1}\left(t_{1}\right) M^{-1}\left(t_{1}\right)$ $\geqslant t_{1}[10$, c. 25$]$, то

$$
\frac{t_{1}}{\left(M^{*}\right)^{-1}\left(t_{1}\right)} \leqslant \frac{\varphi\left(t_{1} s_{1}\right)}{\varphi\left(s_{1}\right)}=\frac{\psi\left(s_{1}\right) t_{1}}{\psi\left(s_{1} t_{1}\right)} .
$$

Сокрашая данное неравенство на $t_{1}$ и полагая $\left(M^{*}\right)^{-1}\left(t_{1}\right)=t_{2}, \psi\left(s_{1}\right)=s_{2}$, получаем неравенство

$$
\psi^{-1}\left(s_{2}\right) M^{*}\left(t_{2}\right) \leqslant \psi^{-1}\left(t_{2} s_{2}\right) \quad\left(s_{2} \geqslant 0, \quad 0 \leqslant t_{2} \leqslant 1\right) .
$$

Поскольку $\left(M^{*}\right)^{*}=M$, то требуемьй результат вытекает из леммы 3 . Лемма доказана.

СлеДСТвИЕ. В условиях леммы 4 пространство Мариинкевича $M_{\psi}\left(X, \mathbb{R}^{n}\right)$ $(\ell)_{M}$-субаддитивно.

Лемма 5. Пусть Е есть б-супераддитивное (б-субаддитивное) НИП, $E^{\prime}$ и $\sigma^{\prime}$ - двойственные $к$ Е и $\sigma$ пространства. Тогда $E^{\prime}$ есть $\sigma^{\prime}$-субаддитивное ( $\sigma^{\prime}$-супераддитивное) пространство.

ДокАЗАТЕльство. Пусть $E$ есть $\sigma$-супераддитивное пространство, $v_{i}(i \in \mathbb{Z})$ - дизъюнктная последовательность функций из $E^{\prime}, v=\sum v_{i}(i \in \mathbb{Z}), b_{i}=\left\|v_{i} ; E^{\prime}\right\|$, $b=\left(b_{i}\right), u \in E,\|u ; E\| \leqslant 1$. Будем считать, что $b \in \sigma^{\prime}$. Обозначим через $X_{i}$ носитель $\operatorname{supp}\left|v_{i}\right|$ функции $\left|v_{i}\right|\left[3\right.$, c. 137] и положим $u_{i}=u 1_{X_{i}}, a_{i}=\left\|u_{i} ; E\right\|, a=\left(a_{i}\right)$. Так как $E$ есть $\sigma$-супераддитивное пространство, то $a \in \sigma$ и $\|a ; \sigma\| \leqslant\|u ; E\| \leqslant 1$. Справедливы соотношения

$$
\langle u, v\rangle=\sum_{i \in \mathbb{Z}}\left\langle u_{i}, v_{i}\right\rangle \leqslant a \cdot b \leqslant\|a ; \sigma\|\left\|b ; \sigma^{\prime}\right\| \leqslant\left\|b ; \sigma^{\prime}\right\| .
$$

Отсюда вытекает неравенство $\left\|u ; E^{\prime}\right\| \leqslant\left\|b ; \sigma^{\prime}\right\|$, означающее $\sigma^{\prime}$-субаддитивность пространства $E^{\prime}$.

Вторая часть леммы устанавливается аналогично. Лемма доказана.

Комбинируя лемму 5 с леммами 1-4, можно сформулировать условия супераддитивности (субаддитивности) пространств, двойственных к $H^{\Psi}\left(X, \mathbb{R}^{n}\right)$, $F_{\psi}\left(X, \mathbb{R}^{n}\right)$ и $L^{\Phi}\left(X, \mathbb{R}^{n}\right)$. Например, в условиях леммы 4 пространство Лоренца $\Lambda_{\psi}\left(X, \mathbb{R}^{n}\right)[4$, c. $145-159] \ell_{M^{*-с у п п е р а д д и т и в н о . ~}}$ 
Лемма 6. Пусть $\Phi \in \beta\left(\mathbb{R}^{n}\right), \Psi \in \beta\left(\mathbb{R}^{m}\right) u$

$$
\Phi(t \xi) \Psi(\eta) \geqslant \Phi(\xi) \Psi(t \eta) \quad\left(\xi \in \mathbb{R}^{n}, \eta \in \mathbb{R}^{m}, 0 \leqslant t \leqslant 1\right) .
$$

Тогда $H^{\Psi}\left(X_{1}, \mathbb{R}^{m}\right) \prec L^{\Phi}\left(X, \mathbb{R}^{n}\right)$ для любого БИП $H=H\left(X_{1}\right)$.

ДокАЗАТЕЛЬСТво. Лемма 6 вытекает из лемм 1,2 .

СлеДСтвИЕ. Пусть $\Phi \in \beta\left(\mathbb{R}^{n}\right), \Psi_{p}(\eta)=|\eta|^{p} \quad\left(\eta \in \mathbb{R}^{m}, 1<p<\infty\right)$. Если при каждом $\xi$ из $\mathbb{R}^{n} \backslash\{0\}$ функция $\Phi(s \xi) s^{-p} \quad(s>0)$ убъвает на $\mathbb{R}_{+}=(0, \infty)$, mо $H^{\Psi p}\left(X_{1}, \mathbb{R}^{m}\right) \prec L^{\Phi}\left(X, \mathbb{R}^{n}\right)$ для любого БИП $H=H\left(X_{1}\right)$.

ДокАЗАТЕЛЬСтво. Для доказательства достаточно заметить, что убывание функции $s \rightarrow \Phi(s \xi) s^{-p}$ на $\mathbb{R}_{+}$влечет оценку $(2)$ в случае $\Psi=\Psi_{p}$.

Обозначим через $\beta_{0}\left(\mathbb{R}^{n}\right)$ часть $\beta\left(\mathbb{R}^{n}\right)$, состоящую из функций $\Phi: \mathbb{R}^{n} \rightarrow \mathbb{R}$, удовлетворяюших оценке $\Phi(2 \xi) \leqslant k_{0} \Phi(\xi)\left(\xi \in \mathbb{R}^{n}, k_{0}<\infty\right)$. Как нетрудно видеть, удовлетворяющая оценке (2) функция $\Phi$ входит в класс $\beta_{0}\left(\mathbb{R}^{n}\right)$. Если $\xi^{*}$ - субградиент функции $\Phi$ в точке $\xi$, то [9, с. 230$] \xi \cdot \xi^{*} \leqslant \Phi(2 \xi)-\Phi(\xi) \leqslant k_{0} \Phi(\xi)$. Отсюда следует, что при любом $p>k_{0}$ и $\xi \neq 0$ функция $s \rightarrow \Phi(s \xi) s^{-p}$ убывает на $\mathbb{R}_{+}$. В частности, включение $\Phi \in \beta_{0}\left(\mathbb{R}^{n}\right)$ гарантирует соотношение $H^{\Psi_{p}}\left(X_{1}, \mathbb{R}^{m}\right) \prec L^{\Phi}\left(X, \mathbb{R}^{n}\right)$ при достаточно большом $p$.

3. Пусть $\Omega$ - область в $\mathbb{R}^{n}(n \geqslant 2), P=P\left(\Omega, \mathbb{R}^{n}\right)$ - НИП измеримых относительно $n$-мерной лебеговой меры $\operatorname{mes}_{n}$ функций на $\Omega$ со значениями в $\mathbb{R}^{n}$. Предполагается, что $1_{\mathscr{K}} a \in P\left(\Omega, \mathbb{R}^{n}\right)$ для любого компакта $\mathscr{K} \subset \Omega$ и любого вектора $a$ из $\mathbb{R}^{n}$. Используются обозначения: $\stackrel{\circ}{\operatorname{Lip}}(\Omega)$ - пространство финитных функций, удовлетворяюших условию Липшица в $\Omega, U(\mathscr{K})=\{f \in \stackrel{\circ}{\operatorname{Lip}}(\Omega), f(x) \geqslant 1$ при $x \in \mathscr{K}\}$.

Каждому компакту $\mathscr{K} \subset \Omega$ сопоставим число

$$
c_{P}(\mathscr{K})=\inf \{\|\nabla f ; P\|, f \in U(\mathscr{K})\},
$$

называемое далее $P$-емкостью компакта $\mathscr{K}$. Удобно положить $c_{P}(\varnothing)=0$. В случае $P=L^{p}\left(\Omega, \mathbb{R}^{n}\right)(1 \leqslant p<\infty)$ аналогичные характеристики множеств использовались многими авторами (см., например, [2], [12]-[14]).

В этом пункте в терминах $P$-емкости формулируется критерий справедливости функционального неравенства $(1)$ для всех $f$ из $\stackrel{\circ}{\operatorname{Lip}}(\Omega)$. Ниже $Q=Q(\Omega)-$ НИП измеримых относительно меры $\operatorname{mes}_{n}$ функций. Наименьшую из констант $C$, для которых имеет место оценка (1), обозначим через $C(P, Q)$. При условии $Q \prec P$ постоянную $C(P, Q)$ удается оценить через постоянную $A(P, Q)$, определяемую как минимальную из констант $a_{0}$, для которых имеет место оценка

$$
\left\|1_{\mathscr{K}} ; Q\right\| \leqslant a_{0} c_{P}(\mathscr{K}),
$$

где $a_{0}$ не зависит от компакта $\mathscr{K} \subset \Omega$.

Теорема 1. Пусть $Q \prec P$. Тогда неравенство (1) справедливо в том и только том случае, если $A(P, Q)<\infty ;$ при этом $A(P, Q) \leqslant C(P, Q) \leqslant$ $4 \nu(P, Q) A(P, Q)$, следовательно, функциональное неравенство (1) эквивалентно геометрическому неравенству (3) с некоторой константой $a_{0}$. 
ДоКАЗАТЕЛЬСтво. Пусть имеет место неравенство (1). Если $f \in U(\mathscr{K})$, то $f(x) \geqslant 1(x \in \mathscr{K})$ и $\|f ; Q\| \geqslant\left\|1_{\mathscr{K}} ; Q\right\|$. В силу (1) $\left\|1_{\mathscr{K}} ; Q\right\| \leqslant C\|\nabla f ; P\|$ $(f \in U(\mathscr{K}))$, поэтому минимизируя по всем $f$ из $U(\mathscr{K})$, получаем $\left\|1_{\mathscr{K}} ; Q\right\| \leqslant$ $C c_{P}(\mathscr{K})$. Отсюда вытекает оценка

$$
A(P, Q) \leqslant C(P, Q) .
$$

Предположим, что $A(P, Q)<\infty$. Фиксируем постоянную $\nu>\nu(P, Q)$ и СП $\sigma$, $\tau$, удовлетворяющие условиям: пространство $P \sigma$-супераддитивно, пространство $Q \tau$-субаддитивно, $\sigma \subset \tau$ и норма оператора вложения $J: \sigma \rightarrow \tau$ не превосходит $\nu$. Установим неравенство (1) для произвольной функции $f$ из $\operatorname{Lip}(\Omega)$.

Пусть $N_{i}=\left\{x \in \Omega,|f(x)| \geqslant 2^{i}\right\}(i \in \mathbb{Z}), t_{+}=\max \{0, t\}, \alpha(t)=\min \left\{1, t_{+}\right\}$. Если $N_{i} \neq \varnothing$, то функция $f_{i}(x)=\alpha\left(2^{1-i}\left(|f(x)|-2^{i-1}\right)\right)$ принадлежит $U\left(N_{i}\right)$, поэтому $\left\|\nabla f_{i} ; P\right\| \geqslant c_{P}\left(N_{i}\right)$. Как нетрудно видеть, последовательность функций $y_{i}=2^{i-1} \nabla f_{i}$ дизъюнктна, $\left\|y_{i} ; P\right\| \geqslant 2^{i-1} c_{P}\left(N_{i}\right)$ и $\sum y_{i}=\nabla|f|$. Из проведенных рассуждений вытекает оценка

$$
\begin{aligned}
& \|\nabla f ; P\| \geqslant\|a ; \sigma\|, \quad a=\left(a_{i}\right), \\
& a_{i}=2^{i-1} c_{P}\left(N_{i}\right), \quad i \in \mathbb{Z} .
\end{aligned}
$$

Последовательность функций $w_{i}=2^{i+1}\left(1_{N_{i}}-1_{N_{i+1}}\right)(i \in \mathbb{Z})$ дизъюнктна, $\left\|w_{i} ; Q\right\| \leqslant 2^{i+1}\left\|1_{N_{i}} ; Q\right\|$ и $\sum w_{i} \geqslant|f|$. Поэтому

$$
\begin{array}{ll}
\|f ; Q\| \leqslant\|b ; \tau\|, & b=\left(b_{i}\right), \\
b_{i}=2^{i+1}\left\|1_{N_{i}} ; Q\right\|, & i \in \mathbb{Z} .
\end{array}
$$

В силу определения константы $A(P, Q)$ имеем

$$
\left\|1_{N_{i}} ; Q\right\| \leqslant A(P, Q) c_{P}\left(N_{i}\right)
$$

Объединяя соотношения (4), (5), получаем

$$
\begin{aligned}
\|f ; Q\| & \leqslant\|b ; \tau\| \leqslant \nu\|b ; \sigma\| \leqslant 4 \nu A(P, Q)\|a ; \sigma\| \\
& \leqslant 4 \nu A(P, Q)\|\nabla f ; P\| .
\end{aligned}
$$

Тем самым установлены неравенство (1) и оценка $C(P, Q) \leqslant 4 \nu A(P, Q)$. Ввиду произвольности $\nu>\nu(P, Q)$ справедливо неравенство $C(P, Q) \leqslant 4 \nu(P, Q) A(P, Q)$. Неравенство (3) равносильно оценке $a_{0} \geqslant A(P, Q)$. Теорема доказана.

условие $Q \prec P$ использовалось лишь при доказательстве достаточности оценки (3) для выполнения неравенства (1). Оно не может быть опушено; обсуждение этого вопроса для пространств $L^{p}\left(\Omega, \mathbb{R}^{n}\right)$ содержится в $[2$, с. 107,172$]$.

Остановимся на некоторых следствиях теоремы 1.

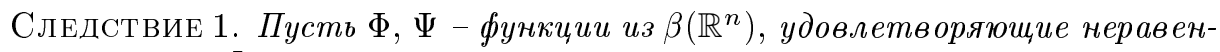
ству (2), $P=L^{\Phi}\left(\Omega, \mathbb{R}^{n}\right), H$ - БИП функций на $\Omega$, измеримых относительно меры mеs $_{n}, Q=H^{\Psi}(\Omega)$. Тогда неравенство (1) справедливо в том и только том случае, если имеет место неравенство (3). 
СлЕДСТвИЕ 2. Пусть $\Phi \in \beta\left(\mathbb{R}^{n}\right), \Phi_{1}: \mathbb{R} \rightarrow \mathbb{R}$ - строго выпуклая четная функция, $\Phi_{1}(0)=0$ и

$$
\Phi(t \xi) \Phi_{1}(s) \geqslant \Phi(\xi) \Phi_{1}(t s) \quad\left(\xi \in \mathbb{R}^{n}, s \geqslant 0,0 \leqslant t \leqslant 1\right)
$$

Пусть $P=L^{\Phi}\left(\Omega, \mathbb{R}^{n}\right), F-$ БИП функций на $\Omega$, измеримых относительно мерьг $\operatorname{mes}_{n}, Q=F_{\psi}$, где $\psi(t)=t / \varphi(t), \varphi: \mathbb{R}_{+} \rightarrow \mathbb{R}_{+}-$функция, обратная $\kappa$ сужению $\Phi_{1}$ на $\mathbb{R}_{+}$. Тогда справедливо заключение следствия 1 .

СлеДСТвИЕ 3. Пусть $1 \leqslant p_{i} \leqslant q_{j} \quad(i, j=1, \ldots, n), P=L_{p_{1} \ldots p_{n}}(\Omega), Q=$ $L_{q_{1} \ldots q_{n}}(\Omega)$ есть пространства со смешанными нормами $[15$, с. 9]. Тогда справедливо заключение следствия 1.

Следствия 1-3 вытекают из теоремы 1 и установленных выше признаков мажорируемости идеальных пространств.

4. Установим непрерывный вариант неравенства (4), близкий к сильным емкостным неравенствам $[2$, с. 98,162$]$. Пусть $V=V\left(\mathbb{R}_{+}, \mu\right)$ - симметричное пространство $[4$, с. 225$]$ функций $v: \mathbb{R}_{+} \rightarrow \mathbb{R}$, измеримых относительно меры $\mu$, инвариантной относительно растяжений и нормированной условием $\mu([1,2])=1$. Дискретизацией $V$ назовем пространство $d V$ таких последовательностей $a=\left(a_{i}\right)$, $i \in \mathbb{Z}$, для которых имеет смысл и конечна норма $\|a ; d V\|=\left\|v_{a} ; V\right\|$, где $v_{a}: \mathbb{R}_{+} \rightarrow \mathbb{R}$ - функция, определенная равенством $v_{a}(t)=a_{i}\left(2^{i} \leqslant t<2^{i+1}, i \in \mathbb{Z}\right)$.

Tеорема 2. Пусть $P=P\left(\Omega, \mathbb{R}^{n}\right)$ есть $d V$-супераддитивное НИП, $f \in$ $\stackrel{\circ}{\operatorname{Lip}}(\Omega), A_{t}=\{x \in \Omega,|f(x)| \geqslant t\}, w(t)=t c_{P}\left(A_{t}\right) \quad\left(t \in \mathbb{R}_{+}\right)$. Тогдa

$$
\|w ; V\| \leqslant 4\|\nabla f ; P\| .
$$

ДокАЗАТЕльСтво. В силу неравенства (4) $\|\nabla f ; P\| \geqslant\|a ; d V\|$, где $a=\left(a_{i}\right)$, $a_{i}=2^{i-1} c_{P}\left(A_{2^{i}}\right), i \in \mathbb{Z}$. Поскольку функция $t \rightarrow c_{P}\left(A_{t}\right)$ убывает на $\mathbb{R}_{+}$, то $4 v_{a}(t) \geqslant w(t)\left(t \in \mathbb{R}_{+}\right)$, следовательно, $4\|a ; d V\|=4\left\|v_{a} ; V\right\| \geqslant\|w ; V\|$. Объединяя эту оценку с (4), приходим к (7). Теорема доказана.

Теорема 1 принимает более обозримую форму в случае, когда $Q=Q(\Omega)$ есть симметричное относительно меры $\operatorname{mes}_{n}$ пространство. В этом случае $\left\|1_{\mathscr{K}} ; Q\right\|=$ $\varphi_{Q}\left(\operatorname{mes}_{n} \mathscr{K}\right)$, где $\varphi_{Q}$ - так называемая фундаментальная функция пространства $Q$ $\left[4\right.$, c. 137]. Сопоставим пространству $P=P\left(\Omega, \mathbb{R}^{n}\right)$ функцию, определенную на интервале $\left(0, m_{0}\right), m_{0}=\operatorname{mes}_{n} \Omega$, равенством $\nu_{P}(t)=\inf \left\{c_{P}(\mathscr{K}), \mathscr{K}\right.$-компактное подмножество области $\left.\Omega, \operatorname{mes}_{n} \mathscr{K}>t\right\}$. При $P=L^{p}\left(\Omega, \mathbb{R}^{n}\right)$ аналогичные $\nu_{P}$ функции изучались в работах [1], [2].

Tеорема 3. Если НИП $P=P\left(\Omega, \mathbb{R}^{n}\right)$ мажорирует $C \Pi Q=Q(\Omega)$, то неравенство (1) әквивалентно неравенству $\varphi_{Q}(t) \leqslant a_{0} \nu_{P}(t) \quad\left(0<t<m_{0}\right.$, $\left.a_{0}<\infty\right)$. 
ДокаЗАТЕЛЬство. Теорема 3 есть следствие теоремы 1.

Приведем оценку функции $\nu_{P}$ в случае, когда $P$ совпадает с пространством Opлича $L^{\Phi}\left(\Omega, \mathbb{R}^{n}\right)$. Напомним некоторые определения. Измеримой по Лебегу функции $f: \Omega \rightarrow \mathbb{R}$ можно сопоставить ее перестановку $\bar{f}$ в убьвающем порядке, определяемую [4, с. 83], [17, с. 332] равенством $\bar{f}(s)=\inf \{t: \mu(f, t) \leqslant s\} \quad\left(0<s<m_{0}\right)$, в котором $\mu(f, t)=\operatorname{mes}_{n}\{x:|f(x)|>t\}$. Если $f \in \stackrel{\circ}{\operatorname{Lip}}(\Omega)$, то функция $\bar{f}$ абсолютно непрерывна на каждом отрезке $[\alpha, \beta] \subset\left(0, m_{0}\right)$ и $\bar{f}(s)=0\left(s \geqslant s_{0}\right.$, $\left.s_{0}<m_{0}\right)$ [18], [19].

Пусть $\Psi: \mathbb{R}^{n} \rightarrow \mathbb{R}$ - выпуклая четная функция, $\Psi(0)=0$ и при любом $h>0$ множество $\left\{\xi \in \mathbb{R}^{n}, \Psi(\xi) \leqslant h\right\}$ компактно и телесно. Функции $\Psi$ можно сопоставить выпуклую четную функцию одного переменного, называемую [19] округлением функции $\Psi$ и характеризуемую условием:

$$
\operatorname{mes}_{n}\left\{\xi \in \mathbb{R}^{n}: \Psi(\xi) \leqslant h\right\}=\operatorname{mes}_{n}\left\{\xi \in \mathbb{R}^{n}, \Psi^{0}(|\xi|) \leqslant h\right\}
$$

при любом $h>0$.

Лемма 7. Пусть $\Phi^{0}$ - округление функиии $\Phi$ из $\beta\left(\mathbb{R}^{n}\right), P=L^{\Phi}\left(\Omega, \mathbb{R}^{n}\right)$, $H=H\left(0, m_{0}\right)$ - двойственное $\kappa L^{\Phi^{0}}\left(0, m_{0}\right)$ пространство. Тогда

$$
\nu_{P}(t) \geqslant\left\|1_{\left[t, m_{0}\right]} \frac{1}{\lambda} ; H\right\|^{-1} \quad\left(0<t<m_{0}\right)
$$

где $\lambda(s)=k_{n} s^{1-1 / n}, k_{n}>0$ - постоянная, зависящая лишь от $n$.

ДокАЗАТЕЛЬСТво основано на вытекающей из [18], [19] оценке

$$
\left\|\nabla f ; L^{\Phi}\right\| \geqslant\left\|\frac{d \bar{f}}{d s} \lambda(s) ; L^{\Phi^{0}}\left(0, m_{0}\right)\right\|
$$

в которой $\bar{f}$ - перестановка функции $f$ из $\stackrel{\circ}{\operatorname{Lip}}(\Omega)$. Пусть $\mathscr{K}$ - компактное подмножество $\Omega, \operatorname{mes}_{n} \mathscr{K}>t>0, \bar{f}$ - перестановка функции $f$ из $U(\mathscr{K})$ и $\bar{f}(s)=0$ при $s \geqslant s_{0}$. Справедливы соотношения

$$
\begin{aligned}
1 \leqslant \bar{f}(t)-\bar{f}\left(s_{0}\right) & =\int_{t}^{s_{0}}\left(-\frac{d \bar{f}}{d s}\right) d s \\
& \leqslant\left\|1_{\left[t, m_{0}\right]} \frac{1}{\lambda} ; H\right\|\left\|\frac{d \bar{f}}{d s} \lambda ; L^{\Phi^{0}}\left(0, m_{0}\right)\right\| \\
& \leqslant\left\|1_{\left[t, m_{0}\right]} \frac{1}{\lambda} ; H\right\|\left\|\nabla f ; L^{\Phi}\right\| .
\end{aligned}
$$

Здесь последовательно используются неравенство $\bar{f}(t) \geqslant 1$, вытекающее из определения $\bar{f}$ и оценки $\operatorname{mes}_{n} \mathscr{K}>t$, равенство $\bar{f}\left(s_{0}\right)=0$, формула Ньютона-Лейбница, применяемая к абсолютно непрерывной на отрезке $\left[t, s_{0}\right]$ функции $\bar{f}$, неравенство Гёльдера для НИП и оценка (9). Таким образом,

$$
\left\|\nabla f ; L^{\Phi}\right\| \geqslant\left\|1_{\left[t, m_{0}\right]} \frac{1}{\lambda} ; H\right\|^{-1} \quad\left(0<t<m_{0}\right)
$$


Минимизируя по всем функциям $f$ из $U(\mathscr{K})$ и компактньм множествам $\mathscr{K} \subset \Omega$, приходим к оценке (8). Лемма доказана.

В работах [18], [19] содержатся варианты оценки (9), возникающие при замене пространства Орлича произвольным симметричным пространством векторных функций. На их основе можно сформулировать соответствующие обобщения леммы 7.

Возврашаясь к обсуждению теоремы 3 , заметим, что близкие к ней утверждения верны и для некоторых пространств со смешанной нормой. В частности, если $P=L_{p_{1} \ldots p_{n}}(\Omega), Q=L_{q_{1} \ldots q_{n}}(\Omega)$, то при условиях $q_{m+1}=\cdots=q_{n}=\infty$, $q_{j}=q \geqslant p_{i} \geqslant 1(j=1, \ldots, m, i=1, \ldots, n)$ неравенство (1) равносильно неравенству $\operatorname{mes}_{m}^{1 / q}(\Pi \mathscr{K}) \leqslant a_{0} c_{P}(\mathscr{K})$, в котором $\Pi$ - оператор ортогонального проектирования $\mathbb{R}^{n}$ на пространство $\mathbb{R}^{m}=\left\{x=\left(x_{1}, \ldots, x_{n}\right), x_{m+1}=\cdots=x_{n}=0\right\}$. Данное замечание легко распространяется на более общие пространства и нелинейные операторы проектирования. Оно предоставляет интерес при сравнении $P$-емкостей с наглядными геометрическими характеристиками компактов типа хаусдорфовых и поперечных мер [2], [20].

5. Далее изучаются общие свойства $P$-емкости. Как полагает автор, эти свойства полезны не только в связи с применениями теоремы 1 к теории вложения, но и при исследовании краевых и вариационных задач с нелинейностями нестепенного характера.

Напомним некоторые определения теории емкостей [12]-[14]. Пусть $\mathfrak{K}(\Omega)$ - совокупность компактных подмножеств отделимого топологического пространства $\Omega$, $\mathfrak{M}(\Omega)$ - система всех подмножеств множества $\Omega$. Емкостью Шоке в $\Omega$ называют функцию $\mathfrak{C}: \mathfrak{K}(\Omega) \rightarrow \mathbb{R}$, удовлетворяюшую условиям:

1) если $\mathscr{K}_{i} \in \mathfrak{K}(\Omega)(i=1,2)$ и $\mathscr{K}_{2} \subset \mathscr{K}_{1}$, то $\mathfrak{C}\left(\mathscr{K}_{2}\right) \leqslant \mathfrak{C}\left(\mathscr{K}_{1}\right)$;

2) для каждого компакта $\mathscr{K}_{0} \subset \Omega$ и числа $\varepsilon>0$ сушествует такая окрестность $G$ компакта $\mathscr{K}_{0}$, что из включений $\mathscr{K} \in \mathfrak{K}(\Omega), \mathscr{K}_{0} \subset \mathscr{K} \subset G$ вытекает неравенство $\mathfrak{C}(\mathscr{K})-\mathfrak{C}\left(\mathscr{K}_{0}\right)<\varepsilon$

3) для любых $\mathscr{K}_{1}, \mathscr{K}_{2}$ из $\mathfrak{K}(\Omega)$ вьполняется неравенство

$$
\mathfrak{C}\left(\mathscr{K}_{1} \cup \mathscr{K}_{2}\right)+\mathfrak{C}\left(\mathscr{K}_{1} \cap \mathscr{K}_{2}\right) \leqslant \mathfrak{C}\left(\mathscr{K}_{1}\right)+\mathfrak{C}\left(\mathscr{K}_{2}\right) .
$$

Условия 1)-3) именуют аксиомами возрастания, непрерывности справа и сильной субаддитивности [13, с. 64]. Обобщенной емкостью в $\Omega$ называют [13, с. 71] функцию $c: \mathfrak{M}(\Omega) \rightarrow \overline{\mathbb{R}}$, удовлетворяющую условиям:

I) если $A_{1} \subset A_{2} \subset \Omega$, то $c\left(A_{1}\right) \leqslant c\left(A_{2}\right)$;

II) для любой возрастаюшей последовательности $A_{i}(i=1,2, \ldots)$ подмножеств $\Omega$ выполняется равенство $c\left(\cup A_{i}\right)=\sup c\left(A_{i}\right)$;

III) для любой убьвающей последовательности $\mathscr{K}_{i}(i=1,2, \ldots)$ компактов из $\Omega$ выполняется равенство $c\left(\cap \mathscr{K}_{i}\right)=\inf c\left(\mathscr{K}_{i}\right)$.

Ниже $\Omega$ - область в $\mathbb{R}^{n}$ с относительной топологией, $\Phi \in \beta\left(\mathbb{R}^{n}\right)$. На множестве $\mathfrak{K}(\Omega)$ определим функции $\mathfrak{C}, \gamma$ равенствами

$$
\begin{aligned}
& \mathfrak{C}(\mathscr{K})=\inf \left\{I_{\Phi}(\nabla f), f \in U(\mathscr{K})\right\}, \\
& \gamma(\mathscr{K})=\inf \left\{\left\|\nabla f ; L^{\Phi}\right\|, f \in U(\mathscr{K})\right\} .
\end{aligned}
$$


Величины $\mathfrak{C}(\mathscr{K}), \gamma(\mathscr{K})$ представляют характеристики массивности компакта $\mathscr{K}$. Первая из них может быть изучена стандартньми для теории емкостей методами.

Лемма 8. Функиия $\mathfrak{C}: \mathfrak{K}(\Omega) \rightarrow \mathbb{R}$ есть емкость Шоке.

ДокАЗАтельство. Аксиома возрастания очевидна. Для доказательства непрерывности справа фиксируем $\mathscr{K}_{0}$ из $\mathfrak{K}(\Omega), \varepsilon>0$ и функцию $f$ из $U\left(\mathscr{K}_{0}\right)$, для которой $I_{\Phi}(\nabla f)<\mathfrak{C}\left(\mathscr{K}_{0}\right)+\varepsilon$. Пусть $\alpha(t)=\min \left\{1, t_{+}\right\}, \lambda_{m}(t)=\alpha((1+1 / m) t)$ $(m=1,2, \ldots ; t \in \mathbb{R}), f_{m}=\lambda_{m} \circ f$. Очевидно, $f_{m} \in \stackrel{\circ}{\operatorname{Lip}}(\Omega), I_{\Phi}\left(\nabla f_{m}\right)<\mathfrak{C}\left(\mathscr{K}_{0}\right)+\varepsilon$ при больших $m\left(m \geqslant m_{0}\right)$, функция $f_{m}$ равна 1 на некоторой окрестности $\mathscr{G}$ компакта $\mathscr{K}_{0}$. Если $\mathscr{K} \in \mathscr{K}(\Omega), \mathscr{K}_{0} \subset \mathscr{K} \subset \mathscr{G}$, то $f_{m} \in U(\mathscr{K})$, поэтому $\mathfrak{C}(\mathscr{K}) \leqslant$ $I_{\Phi}\left(\nabla f_{m}\right)<\mathfrak{C}\left(\mathscr{K}_{0}\right)+\varepsilon$. Непрерывность справа функции $\mathfrak{C}$ доказана.

Пусть $\mathscr{K}_{i} \in \mathfrak{K}(\Omega), f_{i} \in U\left(\mathscr{K}_{i}\right)(i=1,2)$. Тогда функции $g_{1}=\max \left\{f_{1}, f_{2}\right\}$, $g_{2}=\min \left\{f_{1}, f_{2}\right\}$ принадлежат $U\left(\mathscr{K}_{1} \cup \mathscr{K}_{2}\right)$ и $U\left(\mathscr{K}_{1} \cap \mathscr{K}_{2}\right)$, соответственно. Сильная субаддитивность функции $\mathfrak{C}$ обычным образом [1], [12] вытекает из равенства $I_{\Phi}\left(\nabla g_{1}\right)+I_{\Phi}\left(\nabla g_{2}\right)=I_{\Phi}\left(\nabla f_{1}\right)+I_{\Phi}\left(\nabla f_{2}\right)$. Лемма доказана.

Введем аналоги внутренней и внешней емкостей [13, с. 68-70], порождаемых функциями $\mathfrak{C}$ и $\gamma$. Положим

$$
\mathfrak{C}_{*}(A)=\sup \{\mathfrak{C}(\mathscr{K}), \mathscr{K} \subset A\}, \quad \gamma_{*}(A)=\sup \{\gamma(\mathscr{K}), \mathscr{K} \subset A\},
$$

считаем $\mathfrak{C}_{*}(\varnothing)=\gamma_{*}(\varnothing)=0 ;$ пусть

$$
\mathfrak{C}^{*}(A)=\inf \left\{\mathfrak{C}_{*}(\mathscr{G}), \mathscr{G} \supset A\right\}, \quad \gamma^{*}(A)=\inf \left\{\gamma_{*}(\mathscr{G}), \mathscr{G} \supset A\right\},
$$

где инфимум берется по всем открытым множествам $\mathscr{G} \subset \Omega$, содержащим $A$. Из теории емкостей Шоке [12]- [14] следует, что функция $\mathfrak{C}^{*}: \mathfrak{M}(\Omega) \rightarrow \overline{\mathbb{R}}$ есть обобшенная емкость и $\mathfrak{C}^{*}(\mathscr{K})=\mathfrak{C}(\mathscr{K})(\mathscr{K} \in \mathfrak{K}(\Omega))$.

Лемма 9. 1) Если $\gamma_{*}(A)<1$, mo $\mathfrak{C}_{*}(A) \leqslant \gamma_{*}(A)$.

2) $\operatorname{Ecлu} \gamma^{*}(A)<1, m o \mathfrak{C}^{*}(A) \leqslant \gamma^{*}(A)$.

3) $\operatorname{Eсли~} \mathfrak{C}^{*}(A)<1, \operatorname{mo} \gamma^{*}(A) \leqslant 1$.

ДокаЗАТЕЛЬСтво. Пусть $\gamma_{*}(A)<\delta<1$. Для любого компакта $\mathscr{K} \subset A$ найдется функция $f$ из $U(\mathscr{K})$, для которой $I_{\Phi}(\nabla f / \delta)<1$. Так как $I_{\Phi}(\nabla f) \leqslant$ $\delta I_{\Phi}(\nabla f / \delta)<\delta$, то $\mathfrak{C}(\mathscr{K})<\delta$. Следовательно, $\mathfrak{C}_{*}(A) \leqslant \delta$, что доказывает утверждение 1).

Если $\gamma^{*}(A)<\delta<1$, то сушествует открытое множество $\mathscr{G} \supset A, \mathscr{G} \subset \Omega$, для которого $\gamma_{*}(\mathscr{G})<\delta$. В силу уже доказанного $\mathfrak{C}_{*}(\mathscr{G}) \leqslant \gamma_{*}(\mathscr{G})<\delta$, поэтому $\mathfrak{C}^{*}(A) \leqslant$ $\mathfrak{C}_{*}(\mathscr{G})<\delta$, т.е. утверждение 2$)$ также верно.

Пусть $\mathfrak{C}^{*}(A)<1$. Найдется такое открытое множество $\mathscr{G} \supset A, \mathscr{G} \subset \Omega$, что $\mathfrak{C}_{*}(\mathscr{G})<\delta<1$. Для каждого компакта $\mathscr{K} \subset \mathscr{G}$ сушествует функция $f$ из $U(\mathscr{K})$, для которой $I_{\Phi}(\nabla f)<\delta$. Но тогда $\gamma(\mathscr{K}) \leqslant 1 \forall \mathscr{K} \subset \mathscr{G}$, поэтому $\gamma^{*}(A) \leqslant$ $\gamma_{*}(\mathscr{G}) \leqslant 1$. Лемма доказана.

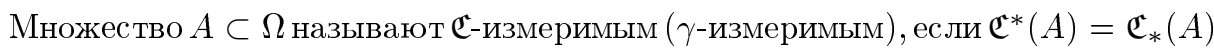
$\left(\gamma^{*}(A)=\gamma_{*}(A)\right)$. Поскольку $\mathfrak{C}^{*}$ есть обобщенная емкость, то (см., например, [12], $[13$, c. $71-74],\left[14\right.$, с. 11]) все аналитические множества $\mathfrak{C}$-измеримы. Через $\mathfrak{C}_{\lambda}^{*}, \mathfrak{C}_{*}^{\lambda}$ ниже обозначаются аналоги функций $\mathfrak{C}^{*}, \mathfrak{C}_{*}$, возникающие при замене функции $\Phi(\xi)$ функцией $\Phi_{\lambda}(\xi)=\Phi(\lambda \xi)(\lambda>0)$. 


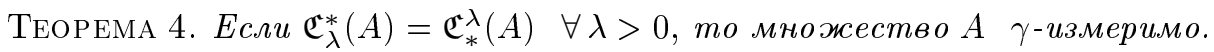

ДокаЗАтЕльСтво. Предположим, что $\mathfrak{C}_{\lambda}^{*}(A)=\mathfrak{C}_{*}^{\lambda}(A) \forall \lambda>0$, но $\gamma_{*}(A)<$ $\gamma^{*}(A)$. Заменяя в случае необходимости функцию $\Phi$ функцией $\Phi_{\lambda}$, можно добиться выполнения неравенств $\gamma_{*}(A)<1<\gamma^{*}(A)$. В силу леммы $9 \mathfrak{C}_{*}(A) \leqslant \gamma_{*}(A)<1$. Но тогда $\mathfrak{C}^{*}(A)=\mathfrak{C}_{*}(A)<1$ и согласно лемме $9 \gamma^{*}(A) \leqslant 1$. Противоречие влечет равенство $\gamma^{*}(A)=\gamma_{*}(A)$. Теорема доказана.

Теорема 5. Функция $\gamma^{*}: \mathfrak{M}(\Omega) \rightarrow \mathbb{R}$ есть обобщенная емкость; если $B_{i} \subset \Omega$ $(i=1,2, \ldots), m o$

$$
\gamma^{*}\left(\bigcup_{i=1}^{\infty} B_{i}\right) \leqslant \sum_{i=1}^{\infty} \gamma^{*}\left(B_{i}\right)
$$

ДокАЗАТЕЛЬСТво. Проверим условия I)-III). Условие I) вьполнено очевидньм образом. Для проверки условия ІІ) фиксируем возрастаюшую последовательность множеств $A_{i} \subset \Omega(i=1,2, \ldots)$. Если $A=\bigcup A_{i}$, то $A_{i} \subset A, \gamma^{*}\left(A_{i}\right) \leqslant \gamma^{*}(A)$, поэтому $\sup \gamma^{*}\left(A_{i}\right) \leqslant \gamma^{*}(A)$.

Предположим, что $\sup \gamma^{*}\left(A_{i}\right)<\gamma^{*}(A)$. Без ограничения обшности считаем, что $\gamma^{*}\left(A_{i}\right)<\delta<1<\gamma^{*}(A)$. В силу леммы $9 \mathfrak{C}^{*}\left(A_{i}\right) \leqslant \delta \forall i$, а поскольку $\mathfrak{C}^{*}$ есть обобшенная емкость, то $\mathfrak{C}^{*}(A) \leqslant \delta<1$. Снова используя лемму 9 , получаем $\gamma^{*}(A) \leqslant 1$. Противоречие влечет II).

Пусть $\mathscr{K}_{i} \in \mathfrak{K}(\Omega)(i=1,2, \ldots), \mathscr{K}_{1} \supset \mathscr{K}_{2} \supset \ldots \supset \mathscr{K}_{i} \supset \ldots$ и $F=\cap \mathscr{K}_{i}$. Тогда $F \subset \mathscr{K}_{i}$, поэтому $\gamma^{*}(F) \leqslant \inf \gamma^{*}\left(\mathscr{K}_{i}\right)$. Если $\gamma^{*}(F)<\inf \gamma^{*}\left(\mathscr{K}_{i}\right)$, то, не уменьшая обшности, можно считать, что $\gamma^{*}(F)<\delta<1<\gamma^{*}\left(\mathscr{K}_{i}\right)$ (в противном случае можно заменить функцию $\Phi$ функцией $\left.\Phi_{\lambda}\right)$. Согласно лемме $9 \mathfrak{C}^{*}(F) \leqslant \gamma^{*}(F)<\delta$. Поскольку $\mathfrak{C}^{*}(F)=\mathfrak{C}(F)$ и $\mathfrak{C}$ есть емкость Шоке, то сушествует такая окрестность $\mathscr{G}$ компакта $F$, что $\mathfrak{C}(\mathscr{K})<\delta$ для любого компакта $\mathscr{K} \subset \mathscr{G}, F \subset \mathscr{K}$. Так как $F=\bigcap \mathscr{K}_{i}$, то $\mathscr{K}_{i} \subset \mathscr{G} \forall i \geqslant i_{0}$. Следовательно, $\mathfrak{C}\left(\mathscr{K}_{i}\right)<\delta \forall i \geqslant i_{0}$ и в силу леммы 9 $\gamma^{*}\left(\mathscr{K}_{i}\right) \leqslant 1 \forall i \geqslant i_{0}$. Полученное противоречие влечет III). Первое утверждение теоремы доказано.

Доказательство неравенства (10) достаточно провести в случае, когда ряд в правой части (10) сходится. Фиксируем $\varepsilon>0$. Для каждого натурального числа найдем открытое множество $\mathscr{G}_{i} \supset B_{i}$, удовлетворяюшее требованию $\gamma_{*}\left(\mathscr{G}_{i}\right)<$ $\gamma^{*}\left(B_{i}\right)+\varepsilon / 2^{i}$. Если компакт $\mathscr{K}$ принадлежит $\bigcup \mathscr{G}_{i}$, то $\mathscr{K} \subset \bigcup_{i=1}^{N} \mathscr{G}_{i}$ при некотором $N$. Поэтому найдутся такие компакты $\mathscr{K}_{1}, \ldots, \mathscr{K}_{N}$, что $\mathscr{K}_{i} \subset \mathscr{G}_{i}(i=1, \ldots, N)$ и $\mathscr{K}=\bigcup_{i=1}^{N} \mathscr{K}_{i}[13$, с. 68$]$. Фиксируем функцию $f_{i}$ из $U\left(\mathscr{K}_{i}\right)$, для которой

$$
\left\|\nabla f_{i} ; L^{\Phi}\right\|<\gamma\left(\mathscr{K}_{i}\right)+\frac{\varepsilon}{2^{i}} \quad(i=1, \ldots, N) .
$$

Функция $f=\left|f_{1}\right|+\cdots+\left|f_{N}\right|$ принадлежит $U(\mathscr{K})$. Следовательно,

$$
\begin{aligned}
\gamma(\mathscr{K}) & \leqslant\left\|\nabla f ; L^{\Phi}\right\| \leqslant \sum_{i=1}^{N}\left\|\nabla\left|f_{i}\right| ; L^{\Phi}\right\|<\sum_{i=1}^{N}\left(\gamma\left(\mathscr{K}_{i}\right)+\frac{\varepsilon}{2^{i}}\right) \\
& \leqslant \sum_{i=1}^{N}\left(\gamma_{*}\left(\mathscr{G}_{i}\right)+\frac{\varepsilon}{2^{i}}\right)<\sum_{i=1}^{\infty} \gamma^{*}\left(B_{i}\right)+2 \varepsilon .
\end{aligned}
$$


Ввиду произвольности компакта $\mathscr{K} \subset \mathscr{G}$ справедлива оценка

$$
\gamma_{*}(\mathscr{G})<\sum_{i=1}^{\infty} \gamma^{*}\left(B_{i}\right)+2 \varepsilon
$$

Так как $\bigcup B_{i} \subset \mathscr{G}$, то из последней оценки вытекает неравенство (10). Теорема доказана.

Каждая из теорем 4, 5 влечет $\gamma$-измеримость аналитических множеств. Обе теоремы без труда переносятся на случай “переменной” функции $\Phi=\Phi(x, \xi)$ $[11$, c. 202]. Аналогичное замечание относится к основньм результатам пунктов 2,3 . Такого рода обобщения представляют интерес для приложений к вариационньм и краевым задачам с анизотропными и вырождаюшимися нелинейностями.

Неравенство (10) выражает свойство полуаддитивности функции $\gamma^{*}$. Остановимся на одном его обобшении.

ТЕОРема 6. Пусть $H=H\left(\Omega, \mathbb{R}^{n}\right)$ есть $\ell_{p}$-субаддитивное НИП $(1 \leqslant p<\infty)$, $c: \mathfrak{M}(\Omega) \rightarrow \overline{\mathbb{R}}$ - внешняя емкость, определяемая как стандартное продолжение на $\mathfrak{M}(\Omega)$ H-емкости $c_{H}$. Пусть $B_{i} \subset \Omega, b_{i}=c\left(B_{i}\right), b=\left(b_{i}\right), B=\bigcup B_{i}$, $i=1,2, \ldots$. Тогда

$$
c(B) \leqslant\left\|b ; \ell_{p}\right\| .
$$

ДокаЗАТЕЛЬСТво. Пусть $\varepsilon>0, \mathscr{K}_{i} \in \mathfrak{K}(\Omega), f_{i} \in U\left(\mathscr{K}_{i}\right)$ и $\left\|\nabla f_{i} ; H\right\|<$ $c_{H}\left(\mathscr{K}_{i}\right)+\varepsilon(i=1,2)$. Положим

$$
f=\max \left\{f_{1}, f_{2}\right\}, \quad D=\left\{x \in \Omega: f_{1}(x) \geqslant f_{2}(x)\right\}, \quad \mathscr{G}=\Omega \backslash D .
$$

Тогда $f \in U\left(\mathscr{K}_{1} \cup \mathscr{K}_{2}\right), \nabla f=1_{D} \nabla f_{1}+1 \mathscr{G} \nabla f_{2} . \quad$ Функции $1_{D} \nabla f_{1}, 1_{\mathscr{G}} \nabla f_{2}$ дизъюнктны, следовательно,

$$
\begin{aligned}
c_{H}\left(\mathscr{K}_{1} \cup \mathscr{K}_{2}\right) & \leqslant\|\nabla f ; H\| \leqslant\left(\left\|\nabla f_{1} ; H\right\|^{p}+\left\|\nabla f_{2} ; H\right\|^{p}\right)^{1 / p} \\
& \leqslant\left(c_{H}^{p}\left(\mathscr{K}_{1}\right)+c_{H}^{p}\left(\mathscr{K}_{2}\right)\right)^{1 / p}+\sqrt[p]{2} \varepsilon .
\end{aligned}
$$

Устремляя $\varepsilon$ к нулю и используя метод математической индукции, получаем для произвольных компактов $\mathscr{K}_{1}, \ldots, \mathscr{K}_{N}$ неравенство

$$
c_{H}\left(\mathscr{K}_{1} \cup \cdots \cup \mathscr{K}_{N}\right) \leqslant\left(c_{H}^{p}\left(\mathscr{K}_{1}\right)+\cdots+c_{H}^{p}\left(\mathscr{K}_{N}\right)\right)^{1 / p} .
$$

Если компакт $\mathscr{K}$ содержится в объединении последовательности открытых множеств $B_{i}(i=1,2, \ldots)$, то найдутся такие компакты $\mathscr{K}_{1}, \ldots, \mathscr{K}_{N}$, что $\mathscr{K}_{i} \subset B_{i}$ $(i=1, \ldots, N)$ и $\mathscr{K}=\mathscr{K}_{1} \cup \cdots \cup \mathscr{K}_{N}[13$, c. 68]. Отсюда вытекает (11) для произвольных открытых множеств $B_{i}(i=1,2, \ldots)$. Общий случай получается теперь путем внешней аппроксимации произвольных множеств открытыми. Теорема доказана.

Суммируем элементы новизны статьи: 1) введено и изучено понятие мажорируемости для НИП; 2) исследованы ранее неизвестные характеристики компактов; 3) установлены новые емкостные неравенства; 4) расширен класс пространств, для которых функциональные неравенства типа теорем вложения эквивалентны геометрическим неравенствам. Общие результаты позволяют получить принципиально новые теоремы вложения для пространств со смешанной нормой и анизотропных пространств Орлича. 


\section{Список литературы}

1. Мазья В. Г. О некоторых интегральных неравенствах для функций многих переменных // Проблемы мат. анализа. №3. Л.: Изд-во ЛГУ, 1972. С. 33-68.

2. Мазья В. Г. Пространства С. Л. Соболева. Л.: Изд-во ЛГУ, 1985.

3. Канторович Л. В., Акилов Г. П. Функциональнй анализ. М.: Наука, 1977.

4. Крейн С.Г., Петунин Ю.И., Семенов Е.М. Интерполяция линейных операторов. М.: Наука, 1978.

5. Брудный Ю.А., Крейн С. Г., Семенов Е. М. Интерполяция линейных операторов // Итоги науки и техники. Математический анализ. Т. 24. М.: ВИНИТИ, 1986. С. 3-163.

6. Митягин Б. С., Швари А. С. Функторы в категориях банаховых пространств // УМН. 1964. Т. 19. № 2. С. $65-130$.

7. Забрейко П. П. Идеальные пространства вектор-функций // Докл. АН БССР. 1987. T. 31. № 4. C. 298-301.

8. Забрейко П.П., Нгуен Хонг Тхай. Теория двойственности идеальных пространств вектор-функций // ДАН СССР. 1990. Т. 311. №6. С. 1296-1299.

9. Рокафеллар Р. Т. Выпуклый анализ. М.: Мир, 1973.

10. Красносельский M. А., Рутицкий $Я$. Б. Вьпуклые функции и пространства Орлича. М.: Физматгиз, 1958.

11. Левин $B$. Л. Выпуклый анализ в пространствах измеримых функций и его применение в математике и экономике. М.: Наука, 1985.

12. Choquet G. Theory of capacities // Ann. Inst. Fourier. 1953. V. 5. P. 131-295.

13. Брело М. Основы классической теории потенциала. М.: Мир, 1964.

14. Карлесон Л. Избранные проблемы теории исключительных множеств. М.: Мир, 1971.

15. Бесов О. В., Ильин В. П., Никольский С. М. Интегральные представления функций и теоремы вложения. М.: Наука, 1975.

16. Соболев С. Л. Введение в теорию кубатурных формул. М.: Наука, 1974.

17. Харди Г. Г., Литтлвуд Д. Е., Полиа Г. Неравенства. М.: ИЛ, 1948.

18. Климов В. С. О перестановках дифференцируемых функций // Матем. заметки. 1971. T. 9. №6. C. $629-638$.

19. Климов В. С. Теоремы вложения и геометрические неравенства // Изв. АН СССР. Сер. матем. 1976. Т. 40. №3. С. 645-671.

20. Бураго Ю. Д., Залгаллер В. А. Геометрические неравенства. Л.: Наука, 1980.

Орловский государственный

Поступила в редакцию

педагогический институт

09.12.1994 